\title{
Effectiveness of Therapeutic Ultrasound with and without Mulligan Mobilzation in Lateral Epicondylitis
}

\author{
Muhammad Waqar Afzal, ${ }^{1}$ Ashfaq Ahmad, ${ }^{2}$ Muhammad Sharif Waqas, ${ }^{3}$ Umair Ahmad ${ }^{4}$
}

\begin{abstract}
Background and Introduction: Mechanical malfunction of elbow joint is the usual cause of lateral elbow pain. Even though varied procedures have suggested for maximizing elbow movements and decreasing elbow pain but mobilization of joints has validated the most efficacious technique. The outcome of manual therapy procedure i.e. Mulligan Mobilization along with Therapeutic ultrasound in lateral epicondylitis to reduce pain and recovery of function had been assimilated in this recent research.
\end{abstract}

Methods: Study is true experimental in nature, randomized control trial adopted to select the subjects with lateral epicondylitis. Thirty patients 15 in each group having LE were chosen Simple random sampling technique and arranged into two groups as guided by CONSORT (Consolidated Standards of Reporting

Conflict of Interest: No

Funding Source: No

Afzal M.W. ${ }^{1}$

Physiotherapy Lecturer, University of Lahore, Pakistan

Ahmad A. ${ }^{2}$

Assistant Professor/HOD of Physiotherapy Department

University of Lahore

Waqas M.S. ${ }^{3}$

Senior Physiotherapist, Services Hospital, Lahore

Ahmad U. ${ }^{4}$

Senior Physiotherapist Lecturer

University of Lahore, Pakistan
Trials) guidelines. Group A received Ultrasound therapy with intensity of $1.2 \mathrm{~W} / \mathrm{cm}^{2}$ with pulsed mode (3MHZ) and duration is 5 minutes .Group B received Ultrasound therapy with an intensity of $1.2 \mathrm{~W} / \mathrm{cm}$ with pulsed mode (3MHZ) for 5 minutes and MWM applied on elbow in supine position shoulder positioned as internal rotation, forearm pronation and elbow extension with 10 repetition for 6 seconds with 15 seconds duration of rest. Total 12 sessions was conducted in duration of 4 weeks after including the patient in study. Four questionnaires was asked to fill i.e. one after every 3 sessions Pain and functional activities were evaluated by PRTEE. Repeated measure ANOVA was used to compare the significance at different time intervals.

Results: The results exhibited that The P-value for PRTEE (patient - rated tennis elbow evaluation) in group A using Therapeutic Ultrasound and was after four weeks of treatment which was less than the level of significance 0.05 . So Therapeutic Ultrasound with Mulligan mobilization was more effective than Therapeutic Ultrasound to reduce pain and restoration of function.

Conclusion: It was concluded that combination of mobilization of Mulligan and Therapeutic ultrasound was more effective compared with Therapeutic Ultrasound in and PRTEE scores in sufferers of lateral epicondylitis.

Key Words: Lateral Epicondylitis, Therapeutic Ultrasound, Mulligan mobilization, PRTEE.

\section{Introduction}

Lateral Epicondolytis has many analogous terms in addition to tennis elbow, peritendinitis of the elbow, lateral elbow pain, lateral epicondylalgia, lateral epi- 
condylitis, and tendonitis of the common extensor origin. Lateral Epicondolytis presents with tenderness and pain on the lower end of the humerus known as lateral epicondyle and pain increases when resistance is tested on the extension of the middle finger, wrist and mostly both. ${ }^{1}$ Because of deprivation of adequate understanding related to pathophysiology give rise to a wide range of treatment options in routine physiotherapy practice along with electrotherapeutic modalities with manual therapy techniques and exercise interventions. ${ }^{2}$ Predominantly the dominant arm is more frequently affected with tennis elbow and form 1000 patients almost 4 to 7 present with this condition, the annual occurrence in the general population is of $1-$ $3 \%$ which has increased up to $19 \%$ in the population of $30-60$ years. $^{3}$

The principal site of pathological changes are evident on extensor carpi radialis brevis tendon along with pathological changes are identified at extensor digitorum communis extensor digitorum longus. The small origin of Extensor carpi radilais breivs shares great amount of forces along its tendon with repeated grasping movement. It is implicated that during all the movements of the forearm as being assailable during shearing stresses. The onset of lateral epicondylitis is not affirmed but it is generally recognized that the repetitive micro trauma or overuse is the cause of tennis elbow. ${ }^{4-6}$

Though stereotypically, the extensor carpi radialis brevis is disturbed, although others may comprise along with extensor digitorum and extensor carpi ulnaris. The region of utmost soreness is archetypally a zone just far to the preliminary mark of the forearm's extensor muscles on the lateral epicondyle. Posterior interosseus nerve and superficial radial at the radiocapitellar joint are the extensions of radial nerve. Pericapsular structures entrapped the Posterior interosseus nerve thus cause the tunnel syndrome The mechanism of injury is increased in tension due to overloading on soft tissue around radial head lack of endurance of muscles in forearm. The development of tennis elbow occurs due to repetition of wrist extensors or the highest degree of torque and abrupt increase in activity with improper surface or equipment. ${ }^{4}$

Mobilization with movement is therapeutic manual therapy procedure used to decline the pain and immediate improvement in functional activates and range of motion. ${ }^{7-9}$ Repositioning of positional faults is proposed in the improvement of lateral epicondylitis by Mobilization with movement. In randomized clini- cal trials the effectiveness of manipulative therapy has been corroborated which appears to improve in terms of decrease in pain and more early gain in of functional restoration. MWW is evident to improve pain and functional activities like every day work related to job, dressing activities, washing the clothes, lifting a cup while drinking tea and glass of water these may be integrated into the treatment plan for the individuals coming with tennis elbow. ${ }^{10}$

Most commonly used modality in Physiotherapy practice is' Therapeutic Ultrasound "to treat musculoskeletal and conditions related to sports injuries and overuse syndromes, for example tendinopathy like rotator cuff, medial epicondylitis and other tendon injuries. Tendon healing is promoted by ultrasound because it stimulates collagen synthesis the tendon cells by stimulating cell migration and proliferation that may benefit tendon healing. ${ }^{11-13}$

Therapeutic ultrasound can be used on two different modes continuous and pulsed mode both used for acute and chronic conditions like rotator cuff tendenopathy, tennis elbow and other conditions of musculoskeletal tissues. It is used on two different frequencies on $3 \mathrm{MHz}$ and $1 \mathrm{MHz}$ In practice 1 and $3 \mathrm{MHz}$ are used for deep and superficial tissue injuries respectively. Intensities of therapeutic ultrasound varies from 0.1 to $1.0 \mathrm{~W} / \mathrm{cm}^{2}$ according to tissue depth and type of injury. Duration of application of Therapeutic ultrasound ranges between 2 and 5minutes and applying in circular manner by the physiotherapist. ${ }^{13}$

\section{Material and Methods}

Study Design: Study is true experimental in nature, randomized control trial adopted to select the subjects with lateral epicondylitis.

Setting: Data was collected from Services and Alshafi Hospital Allma Iqbal Town Lahore. It is comparative study design randomized clinical trials. Subjects with lateral epicondylitis in the physiotherapy department.

Duration: It was completed within 6 months after the approval from Technique Review committee.

Sample Size: Sample size of 30 subjects (15 in each) was taken in each group using 5\% level of significance and $80 \%$ power of test.

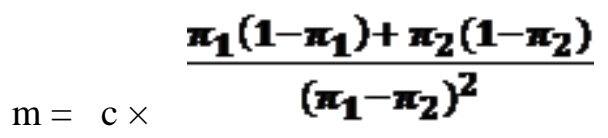


$\mathrm{c}=7.9$ at $80 \%$ power of test

$\pi_{1}=$ Estimated proportion of success of standard therapy.

$\pi_{2}=$ Proportion of total successful outcome.

According to this formula " $\mathrm{m} "=15+15$ (per each group) $=30$.

Sample Technique: Simple random sampling technique was used.

\section{Sample Selection Criteria}

- Inclusion Criteria:

- Referred patients having lateral epicondylitis.

- The age between 20 - 50 years.

- Patient's willingness in participation in this study.

\section{Exclusion Criteria}

- Skin infection.

- Recent trauma to elbow.

- Infection in or surrounding the elbow.

- Any type of peripheral nerve injury or involvement in upper extremity.

- Cervical radiculopathy.

- Stroke.

- Head injury.

- Elbow joint dislocation.

- Fractures of radius humerus and ulna.

- Rheumatoid arthritis.

- Tumors.

\section{Methodology}

Thirty patients was selected randomly and randomly allocated into two groups 15 in each as per Consort Guidelines. ${ }^{14}$ Group A received Ultrasound therapy with intensity of $1.2 \mathrm{~W} / \mathrm{cm}$ with pulsed mode (3MHZ) and duration is 5 minutes.

Group B received Ultrasound therapy with an intensity of $1.2 \mathrm{~W} / \mathrm{cm}$ with pulsed mode (3MHZ) for 5 minutes and MWM applied on elbow in supine position shoulder postioned as internal rotation, forearm pronation and elbow extension with 10 repetition for 6 seconds with 15 seconds duration of rest.

Data Collection Procedure: Thirty patients who completed the selection criteria were enrolled in this study. Written informed consent was taken from every individual participating in this study prior to performing any examination. Allocation of patients in two groups had done by simple random sampling to assure external validity as per CONSORT guidelines 2010. Group A was treated with Ultrasound therapy group B with Ultrasound Therapy Mulligan mobilization.Both groups were received the conventional therapy, which was remained same throughout the study. The conventional therapy was included Ultrasonic Therapy (ITO US-100) with intensity of $1.2 \mathrm{~W} / \mathrm{cm}^{2}$ with pulsed mode ( $3 \mathrm{MHZ}$ ) and duration is 5 minutes.

Group A was received Ultrasound therapy plus Mulligan mobilization applied on elbow in supine position shoulder positioned as internal rotation, forearm pronation and elbow extension with 10 repetition for 6 seconds with 15 seconds duration of rest. Pain intensity and functional activities were appraised by PRTEE. All this information was gathered by using a pre-designed questionnaire as PRTEE. Confounding variables were controlled by randomization and restriction methods. All treatment was applied by single handed for controlling bias.

\section{Results}

There were total 30 cases who were enrolled in this study. The mean age of the cases was $34.33 \pm 8.80$ of which $53 \%$ were male and $47 \%$ were female cases. There were $8(53 \%)$ were male and $7(46 \%)$ were female in group one $7(46 \%)$ male and $8(53 \%)$ female in group 2.and Overall baseline pain score for repeating arm movement was $6.00 \pm 1.50$, when carrying plastic bag was $7.13 \pm 1.38$ and when pain was at its worse was $8.60 \pm 1.27$. Functional disability score was $6.10 \pm 1.62$ in cases who were have issue even in turning the knob of door, $7.13 \pm 1.10$ rate was given by those who carry bag, cases who were noted during opening a jat $7.73 \pm 1.11$ score and wring out washing cloth of towel has $5.33 \pm 1.15$ score. Usual activity score at base line on personal activity, household work, work, recreational sporting was $7.03 \pm 1.82$, $7.00 \pm 1.76,7.06 \pm 1.18$ and $5.23 \pm 1.85$ respectively. There was significant decrease in the pain score, functional ability score and usual activity as per presented in the tables for the both groups (Table 17 to 26). All the $\mathrm{p}$-value for the groups comparison were significant $(\mathrm{p}$ value $<0.005)$. 
Table 1: Baseline Pain Score in different categories.

\begin{tabular}{|l|c|c|c|}
\hline & $\begin{array}{c}\text { Pain: When doing a Task with } \\
\text { Repeated Arm Movement }\end{array}$ & $\begin{array}{c}\text { Pain: When Carrying } \\
\text { Plastic Bag of Groceries }\end{array}$ & $\begin{array}{c}\text { Pain: When your Pain was } \\
\text { at its Worst }\end{array}$ \\
\hline $\mathrm{N}$ & 30 & 30 & 30 \\
\hline Mean & 6.0000 & 7.1333 & 8.6000 \\
\hline Std. Deviation & 1.50860 & 1.38298 & 1.27577 \\
\hline Minimum & 3.00 & 4.00 & 4.00 \\
\hline Maximum & 8.00 & 9.00 & 10.00 \\
\hline
\end{tabular}

Table 2: Baseline Functional Disability Score.

\begin{tabular}{|l|c|c|c|c|}
\hline & $\begin{array}{c}\text { Turn a Doorknob or } \\
\text { Key }\end{array}$ & $\begin{array}{c}\text { Carry a Grocery Bag or } \\
\text { Briefcase by the Handle }\end{array}$ & Open a Jar & $\begin{array}{c}\text { Wring out a Wash } \\
\text { Cloth or Wet Towel }\end{array}$ \\
\hline $\mathrm{N}$ & 30 & 30 & 30 & 30 \\
\hline Mean & 6.1000 & 7.1333 & 7.7333 & 5.3333 \\
\hline Std. Deviation & 1.62629 & 1.10589 & 1.11211 & 1.15470 \\
\hline Minimum & 4.00 & 4.00 & 4.00 & 3.00 \\
\hline Maximum & 9.00 & 9.00 & 9.00 & 8.00 \\
\hline
\end{tabular}

Table 3: Baseline Usual Activity score.

\begin{tabular}{|l|c|c|c|c|}
\hline & $\begin{array}{c}\text { Usual Activities: } \\
\text { Personal Activities } \\
\text { (Dressing, Washing) }\end{array}$ & $\begin{array}{c}\text { Usual Activities: } \\
\text { Household Work } \\
\text { (Cleaning, Maintained }\end{array}$ & $\begin{array}{c}\text { Usual Activities: } \\
\text { Work (Your Job or } \\
\text { Everyday Work) }\end{array}$ & $\begin{array}{c}\text { Usual Activities: } \\
\text { Recreational Orr } \\
\text { Sporting Activities }\end{array}$ \\
\hline $\mathrm{N}$ & 30 & 30 & 30 & 30 \\
\hline Mean & 7.0333 & 7.0000 & 7.0667 & 5.2333 \\
\hline Std. Deviation & 1.82857 & 1.76166 & 4.81817 & 1.95965 \\
\hline Minimum & 4.00 & 4.00 & 9.00 & 3.00 \\
\hline Maximum & 9.00 & 9.00 & 9.00 \\
\hline
\end{tabular}

Table 4: Pain score at $1^{\text {st }}$ Follow-up.

\begin{tabular}{|l|c|c|c|}
\hline & $\begin{array}{c}\text { Pain: When Doing a Task } \\
\text { with Repeated Arm } \\
\text { Movement 1st Follow-up }\end{array}$ & $\begin{array}{c}\text { Pain: When Carrying } \\
\text { Plastic Bag of Groceries } \\
\text { 1st Follow-up }\end{array}$ & $\begin{array}{c}\text { Pain: When Your Pain was } \\
\text { at its Worst 1st Follow-up }\end{array}$ \\
\hline $\mathrm{N}$ & 30 & 30 & 30 \\
\hline Mean & 5.3000 & 6.3000 & 7.5667 \\
\hline Std. Deviation & 1.46570 & .98786 & 1.25075 \\
\hline Minimum & 3.00 & 5.00 & 4.00 \\
\hline Maximum & 8.00 & 8.00 & 9.00 \\
\hline
\end{tabular}


Table 5: Functional disability score at $1^{\text {st }}$ follow-up

\begin{tabular}{|l|c|c|c|c|}
\hline & $\begin{array}{c}\text { Functional } \\
\text { Disability: Turn a } \\
\text { Doorknob or Key } \\
\text { 1st Follow-up }\end{array}$ & $\begin{array}{c}\text { Functional Disabilities: } \\
\text { Carry a Briefcase or } \\
\text { Grocery Bag by the } \\
\text { Handle 1st Follow-up }\end{array}$ & $\begin{array}{c}\text { Functional } \\
\text { Disabilities: Open a } \\
\text { Jar 1st Follow-up }\end{array}$ & $\begin{array}{c}\text { Functional Disability: } \\
\text { Wring out Wet Towel or } \\
\text { Washcloth 1st Follow-up }\end{array}$ \\
\hline $\mathrm{N}$ & 30 & 30 & 30 & 30 \\
\hline Mean & 5.1667 & 5.9667 & 6.9667 & 4.7333 \\
\hline Std. Deviation & 1.26173 & 1.18855 & 1.12903 & 1.14269 \\
\hline Minimum & 3.00 & 3.00 & 4.00 & 2.00 \\
\hline Maximum & 8.00 & 8.00 & 9.00 & 7.00 \\
\hline
\end{tabular}

Table 6: Usual activity score at $1^{\text {st }}$ follow-up.

\begin{tabular}{|l|c|c|c|c|}
\hline & $\begin{array}{c}\text { Usual Activities: } \\
\text { Personal Activities } \\
\text { (Dressing, Washing) } \\
\text { 1st Follow-up }\end{array}$ & $\begin{array}{c}\text { Usual Activities: } \\
\text { Household Work } \\
\text { (Cleaning, Maintained } \\
\text { 1st Follow-up }\end{array}$ & $\begin{array}{c}\text { Usual Activities: } \\
\text { Work (Your Job or } \\
\text { Everyday Work) 1st } \\
\text { Follow-up }\end{array}$ & $\begin{array}{c}\text { Usual Activities: } \\
\text { Recreational or } \\
\text { Sporting Activities } \\
\text { 1st Follow-up }\end{array}$ \\
\hline $\mathrm{N}$ & 30 & 30 & 30 & 30 \\
\hline Mean & 6.1000 & 6.1667 & 6.3000 & 4.7667 \\
\hline Std. Deviation & 1.18467 & 1.55549 & 3.53466 & 1.59056 \\
\hline Minimum & 3.00 & 3.00 & 8.00 & 3.00 \\
\hline Maximum & 8.00 & 8.00 & 8.00 \\
\hline
\end{tabular}

Table 7: Pain score at $2^{\text {nd }}$ Follow-up.

\begin{tabular}{|l|c|c|c|}
\hline & $\begin{array}{c}\text { Pain: When Doing a Task } \\
\text { with Repeated Arm } \\
\text { Movement 2nd Follow-up }\end{array}$ & $\begin{array}{c}\text { Pain: When Carrying a } \\
\text { Plastic Bag of Groceries } \\
\text { 2nd Follow-up }\end{array}$ & $\begin{array}{c}\text { Pain: When Your Pain was } \\
\text { at its Worst 2nd Follow-up }\end{array}$ \\
\hline $\mathrm{N}$ & 30 & 30 & 30 \\
\hline Mean & 4.8333 & 6.1000 & 7.7667 \\
\hline Std. Deviation & 1.14721 & .75886 & .89763 \\
\hline Minimum & 3.00 & 5.00 & 6.00 \\
\hline Maximum & 7.00 & 7.00 & 9.00 \\
\hline
\end{tabular}

Table 8: Functional disability score at $2^{\text {nd }}$ Follow-up.

\begin{tabular}{|l|c|c|c|c|}
\hline & $\begin{array}{c}\text { Functional } \\
\text { Disability: Turn a } \\
\text { Dooknob or Key 2 } \\
\text { Follow-up }\end{array}$ & $\begin{array}{c}\text { Functional Disabilities: } \\
\text { Carry Briefcase or a Grocery } \\
\text { Bag or by the Handle } \\
2^{\text {nd }}\end{array}$ & $\begin{array}{c}\text { Functional } \\
\text { Follow-up } \\
\text { Disabilities: } \\
\text { Open a Jar 2 } \\
\text { Follow-up }\end{array}$ & $\begin{array}{c}\text { Functional Disability: } \\
\text { Wring Out Wet Towel } \\
\text { or a Washcloth or 2 } \\
\text { Follow-up }\end{array}$ \\
\hline $\mathrm{N}$ & 30 & 30 & 30 & 30 \\
\hline Mean & 4.9333 & 5.3333 & 5.5000 & 4.2667 \\
\hline Std. Deviation & 1.38796 & 1.53877 & 1.25258 & 1.04826 \\
\hline Minimum & 2.00 & 2.00 & 3.00 & 2.00 \\
\hline Maximum & 7.00 & 9.00 & 8.00 & 6.00 \\
\hline
\end{tabular}


Table 9: Usual activity score at $2^{\text {nd }}$ Follow-up.

\begin{tabular}{|l|c|c|c|c|}
\hline & $\begin{array}{c}\text { Usual Activities: } \\
\text { Personal Activities } \\
\text { (Dressing, Washing) } \\
\text { 2nd Follow-up }\end{array}$ & $\begin{array}{c}\text { Usual Activities: } \\
\text { Household Work } \\
\text { (Cleaning, Maintenance } \\
\text { 2nd Follow-up }\end{array}$ & $\begin{array}{c}\text { Usual Activities: } \\
\text { Work (Your Job or } \\
\text { Everyday Work) 2nd } \\
\text { Follow-up }\end{array}$ & $\begin{array}{c}\text { Usual Activities: } \\
\text { Recreaional orr } \\
\text { Sporting Activities } \\
\text { 2st Follow-up }\end{array}$ \\
\hline $\mathrm{N}$ & 30 & 30 & 30 & 30 \\
\hline Mean & 5.6000 & 5.1333 & 5.4000 & 4.0000 \\
\hline Std. Deviation & 1.32873 & 1.50249 & 1.40443 & 1.28654 \\
\hline Minimum & 3.00 & 3.00 & 3.00 & 2.00 \\
\hline Maximum & 8.00 & 8.00 & 8.00 & 7.00 \\
\hline
\end{tabular}

Table 10: Pain score at $3^{\text {rd }}$ follow-up.

\begin{tabular}{|c|c|c|c|}
\hline & $\begin{array}{l}\text { Pain: When doing a Task } \\
\text { with Repeated Arm } \\
\text { Movement 3rd Follow-up }\end{array}$ & $\begin{array}{c}\text { Pain: When Carrying a } \\
\text { Plastic Bag of Groceries } \\
\text { 3rd Follow-up }\end{array}$ & $\begin{array}{l}\text { Pain: When Your Pain was } \\
\text { at its Worst 3rd Follow-up }\end{array}$ \\
\hline $\mathrm{N}$ & 30 & 30 & 30 \\
\hline Mean & 3.6333 & 5.6667 & 7.2667 \\
\hline Std. Deviation & .96431 & .80230 & .86834 \\
\hline Minimum & 2.00 & 4.00 & 6.00 \\
\hline Maximum & 5.00 & 7.00 & 9.00 \\
\hline
\end{tabular}

Table 11: Functional disability score at $3^{\text {rd }}$ follow-up.

\begin{tabular}{|l|c|c|c|c|}
\hline & $\begin{array}{c}\text { Functional Disability: } \\
\text { Turn a Doorknob or } \\
\text { Key 3 }\end{array}$ & $\begin{array}{c}\text { Functional Disabilities: } \\
\text { Carry Briefcase or a } \\
\text { Grocery Bag by the } \\
\text { Handle 3 }{ }^{\text {rd }} \text { Follow-up }\end{array}$ & $\begin{array}{c}\text { Functional } \\
\text { Disabilities: } \\
\text { Open a Jar 3 } \\
\text { Follow-up }\end{array}$ & $\begin{array}{c}\text { Functional Disability: } \\
\text { Wring Out Wet Towel } \\
\text { or a Washcloth } \\
3^{\text {rd }} \text { Follow-up }\end{array}$ \\
\hline $\mathrm{N}$ & 30 & 30 & 30 & 30 \\
\hline Mean & 3.7000 & 4.1000 & 5.3667 & 3.7333 \\
\hline Std. Deviation & 1.08755 & 1.53914 & 1.15917 & 1.25762 \\
\hline Minimum & 2.00 & 2.00 & 3.00 & 8.00 \\
\hline Maximum & 6.00 & 8.00 & 2.00 \\
\hline
\end{tabular}

Table 12: Usual activity score at $3^{\text {rd }}$ follow-up.

\begin{tabular}{|l|c|c|c|c|}
\hline & $\begin{array}{c}\text { Usual Activities: } \\
\text { Personal Activities } \\
\text { (Dressing, Washing) } \\
3^{\text {rd }} \text { Follow-up }\end{array}$ & $\begin{array}{c}\text { Usual Activities: } \\
\text { Household Work } \\
\text { (Cleaning, Maintenance } \\
3^{\text {rd }} \text { Follow-up }\end{array}$ & $\begin{array}{c}\text { Usual Activities: } \\
\text { Work (Your Job or } \\
\text { Everyday Work) } \\
3^{\text {rd }} \text { Follow-up }\end{array}$ & $\begin{array}{c}\text { Usual Activities: } \\
\text { Recreational orr } \\
\text { Sporting Activities } \\
3^{\text {rd }} \text { Follow-up }\end{array}$ \\
\hline $\mathrm{N}$ & 30 & 30 & 30 & 30 \\
\hline Mean & 4.9000 & 4.2667 & 4.4000 & 3.0667 \\
\hline Std. Deviation & 1.32222 & 1.04826 & 1.32873 & 1.38796 \\
\hline Minimum & 2.00 & 3.00 & 2.00 & 7.00 \\
\hline Maximum & 8.00 & 6.00 & & 6.00 \\
\hline
\end{tabular}


Table 13: Distribution according to Age.

\begin{tabular}{|l|c|}
\hline \multicolumn{2}{|l|}{ Age of Patients } \\
\hline $\mathrm{N}$ & 30 \\
\hline Mean & 34.3333 \\
\hline Std. Deviation & 8.80569 \\
\hline Minimum & 21.00 \\
\hline Maximum & 48.00 \\
\hline
\end{tabular}

Table 14: Comparison of mean pain when doing a task with repeated arm movement at different followup.

\begin{tabular}{|c|c|c|c|c|}
\hline (I) pain & (2) pain & Mean Difference (I-J) & $\begin{array}{l}\text { Std. } \\
\text { Error }\end{array}$ & Sig. ${ }^{\text {a }}$ \\
\hline \multirow{3}{*}{1} & 2 & $.700^{*}$ & .199 & .001 \\
\hline & 3 & $1.167^{*}$ & .315 & .001 \\
\hline & 4 & $2.367^{*}$ & .327 & .000 \\
\hline \multirow{3}{*}{2} & 1 & $-.700^{*}$ & .199 & .001 \\
\hline & 3 & .467 & .328 & .165 \\
\hline & 4 & $1.667^{*}$ & .350 & .000 \\
\hline \multirow{3}{*}{3} & 1 & $-1.167^{*}$ & .315 & .001 \\
\hline & 2 & -.467 & .328 & .165 \\
\hline & 4 & $1.200^{*}$ & .169 & .000 \\
\hline \multirow{3}{*}{4} & 1 & $-2.367^{*}$ & .327 & .000 \\
\hline & 2 & $-1.667^{*}$ & .350 & .000 \\
\hline & 3 & $-1.200^{*}$ & .169 & .000 \\
\hline
\end{tabular}

Table 15: Comparison of mean pain when carrying a plastic bag of groccessries at different follow up:

\begin{tabular}{|c|c|c|c|c|}
\hline (I) pain & (2) pain & Mean Difference (I-J) & $\begin{array}{l}\text { Std. } \\
\text { Error }\end{array}$ & Sig. ${ }^{\text {a }}$ \\
\hline \multirow{3}{*}{1} & 2 & $.833^{*}$ & .198 & .000 \\
\hline & 3 & $1.033^{*}$ & .265 & .001 \\
\hline & 4 & $1.467^{*}$ & .270 & .000 \\
\hline \multirow{3}{*}{2} & 1 & $-.833^{*}$ & .198 & .000 \\
\hline & 3 & .200 & .169 & .246 \\
\hline & 4 & $.633^{*}$ & .217 & .007 \\
\hline \multirow{3}{*}{3} & 1 & $-1.033^{*}$ & .265 & .001 \\
\hline & 2 & -.200 & .169 & .246 \\
\hline & 4 & $.433^{*}$ & .157 & .010 \\
\hline \multirow{3}{*}{4} & 1 & $-1.467^{*}$ & .270 & .000 \\
\hline & 2 & $-.633^{*}$ & .217 & .007 \\
\hline & 3 & $-.433^{*}$ & .157 & .010 \\
\hline
\end{tabular}

Table 16: Comparison of mean pain when your pain was at worst at different follow-up

\begin{tabular}{|c|c|c|c|c|c|c|}
\hline \multirow{2}{*}{ (I) pain } & \multirow{2}{*}{ (2) pain } & \multirow{2}{*}{ Mean Difference (I-J) } & \multirow{2}{*}{ Std. Error } & \multirow{2}{*}{ Sig. ${ }^{a}$} & \multicolumn{2}{|c|}{$95 \%$ Confidence Interval for Difference } \\
\hline & & & & & Lower Bound & Upper Bound \\
\hline \multirow{3}{*}{1} & 2 & $1.033^{*}$ & .169 & .000 & .687 & 1.380 \\
\hline & 3 & $.833^{*}$ & .235 & .001 & .352 & 1.315 \\
\hline & 4 & $1.333^{*}$ & .205 & .000 & .913 & 1.753 \\
\hline \multirow{3}{*}{2} & 1 & $-1.033^{*}$ & .169 & .000 & -1.380 & -.687 \\
\hline & 3 & -.200 & .206 & .339 & -.621 & .221 \\
\hline & 4 & .300 & .210 & .163 & -.129 & .729 \\
\hline
\end{tabular}


EFFECTIVENESS OF THERAPEUTIC ULTRASOUND WITH AND WITHOUT MULLIGAN MOBILZATION IN LATERAL EPICONDYLITIS

\begin{tabular}{|c|c|c|c|c|c|c|}
\hline \multirow{3}{*}{3} & 1 & $-.833^{*}$ & .235 & .001 & -1.315 & -.352 \\
\cline { 2 - 7 } & 2 & .200 & .206 & .339 & -.221 & .621 \\
\cline { 2 - 7 } & 4 & $.500^{*}$ & .093 & .000 & .310 & .690 \\
\hline \multirow{3}{*}{4} & 1 & $-1.333^{*}$ & .205 & .000 & -1.753 & -.913 \\
\cline { 2 - 7 } & 2 & -.300 & .210 & .163 & -.729 & .129 \\
\cline { 2 - 7 } & 3 & $-.500^{*}$ & .093 & .000 & -.690 & -.310 \\
\hline
\end{tabular}

Table 17: Comparison of mean functional ability score when turning a door knob at different follow-up.

\begin{tabular}{|c|c|c|c|c|}
\hline (I) Functional & (2) Functional & Mean Difference (I-J) & Std. Error & Sig. $^{\text {a }}$ \\
\hline \multirow{3}{*}{1} & 2 & $.933^{*}$ & .271 & .002 \\
\cline { 2 - 5 } & 3 & $1.167^{*}$ & .307 & .001 \\
\cline { 2 - 5 } & 4 & $2.400^{*}$ & .379 & .000 \\
\hline \multirow{3}{*}{2} & 1 & $-.933^{*}$ & .271 & .002 \\
\cline { 2 - 5 } & 3 & .233 & .257 & .371 \\
\cline { 2 - 5 } & 4 & $1.467^{*}$ & .295 & .000 \\
\hline \multirow{3}{*}{3} & 1 & $-1.167^{*}$ & .307 & .001 \\
\cline { 2 - 5 } & 2 & -.233 & .257 & .371 \\
\hline \multirow{3}{*}{4} & 1 & $1.233^{*}$ & .266 & .000 \\
\cline { 2 - 5 } & 2 & $-2.400^{*}$ & .297 & .000 \\
\cline { 2 - 5 } & 3 & $-1.467^{*}$ & .266 & .000 \\
\hline
\end{tabular}

Table 18: Comparison of mean functional ability score when carrying grocery bag by handle at different follow-up

\begin{tabular}{|c|c|c|c|c|}
\hline (I) Functional & (2) Functional & Mean Difference (I-J) & Std. Error & Sig. $^{\text {a }}$ \\
\hline \multirow{4}{*}{1} & 2 & $1.167^{*}$ & .209 & .000 \\
\cline { 2 - 5 } & 3 & $1.800^{*}$ & .363 & .000 \\
\cline { 2 - 5 } & 4 & $3.033^{*}$ & .394 & .000 \\
\hline \multirow{3}{*}{2} & 1 & $-1.167^{*}$ & .209 & .000 \\
\cline { 2 - 5 } & 3 & $.633^{*}$ & .256 & .019 \\
\hline \multirow{3}{*}{3} & 4 & $1.867^{*}$ & .310 & .000 \\
\cline { 2 - 5 } & 1 & $-1.800^{*}$ & .363 & .000 \\
\cline { 2 - 5 } & 2 & $-.633^{*}$ & .256 & .019 \\
\hline \multirow{3}{*}{4} & 4 & $1.233^{*}$ & .302 & .000 \\
\cline { 2 - 5 } & 2 & $-3.033^{*}$ & .310 & .000 \\
\cline { 2 - 5 } & 3 & $-1.867^{*}$ & .302 & .000 \\
\hline
\end{tabular}


Table 19: Comparison of mean functional ability score when opens a jar at different follow-up.

\begin{tabular}{|c|c|c|c|c|}
\hline (I) Functional & (2) Functional & Mean Difference (I-J) & Std. Error & Sig. $^{\text {a }}$ \\
\hline \multirow{4}{*}{1} & 2 & $.767^{*}$ & .184 & .000 \\
\cline { 2 - 5 } & 3 & $2.233^{*}$ & .274 & .000 \\
\cline { 2 - 5 } & 4 & $2.367^{*}$ & .265 & .000 \\
\hline \multirow{3}{*}{2} & 1 & $-.767^{*}$ & .184 & .000 \\
\cline { 2 - 5 } & 3 & $1.467^{*}$ & .287 & .000 \\
\hline \multirow{3}{*}{3} & 4 & $1.600^{*}$ & .282 & .000 \\
\cline { 2 - 5 } & 1 & $-2.233^{*}$ & .274 & .000 \\
\cline { 2 - 5 } & 2 & $-1.467^{*}$ & .287 & .000 \\
\hline \multirow{3}{*}{4} & 4 & .133 & .266 & .620 \\
\cline { 2 - 5 } & 2 & $-2.367^{*}$ & .282 & .000 \\
\cline { 2 - 5 } & 3 & $-1.600^{*}$ & .266 & .620 \\
\hline
\end{tabular}

Table 20: Comparison of mean functional ability score when wring out a washing cloth or towel at different follow-up.

\begin{tabular}{|c|c|c|c|c|}
\hline (I) Functional & (2) Functional & Mean Difference (I-J) & Std. Error & Sig. $^{\text {a }}$ \\
\hline \multirow{4}{*}{1} & 2 & $.600^{*}$ & .207 & .007 \\
\cline { 2 - 5 } & 3 & $1.067^{*}$ & .275 & .001 \\
\cline { 2 - 5 } & 4 & $1.600^{*}$ & .324 & .000 \\
\hline \multirow{3}{*}{2} & 1 & $-.600^{*}$ & .207 & .007 \\
\cline { 2 - 5 } & 3 & .467 & .229 & .050 \\
\hline \multirow{3}{*}{3} & 4 & $1.000^{*}$ & .244 & .000 \\
\cline { 2 - 5 } & 1 & $-1.067^{*}$ & .275 & .001 \\
\cline { 2 - 5 } & 2 & -.467 & .229 & .050 \\
\hline \multirow{3}{*}{4} & 1 & .533 & .270 & .058 \\
\cline { 2 - 5 } & 2 & $-1.600^{*}$ & .324 & .000 \\
\cline { 2 - 5 } & 3 & $-1.000^{*}$ & .244 & .058 \\
\hline
\end{tabular}

Table 21: Comparison of mean usual activity score during personal activity at different follow-up.

\begin{tabular}{|c|c|c|c|c|}
\hline (I) Usual Activity & (2) Usual Activity & Mean Difference (I-J) & Std. Error & Sig. $^{\text {a }}$ \\
\hline \multirow{3}{*}{1} & 2 & $.933^{*}$ & .253 & .001 \\
\cline { 2 - 5 } & 3 & $1.433^{*}$ & .389 & .001 \\
\cline { 2 - 5 } & 4 & $2.133^{*}$ & .403 & .000 \\
\hline
\end{tabular}




\begin{tabular}{|c|c|c|c|c|}
\hline \multirow{3}{*}{2} & 1 & $-.933^{*}$ & .253 & .001 \\
\cline { 2 - 5 } & 3 & .500 & .248 & .053 \\
\cline { 2 - 5 } & 4 & $1.200^{*}$ & .293 & .000 \\
\hline \multirow{3}{*}{3} & 1 & $-1.433^{*}$ & .389 & .001 \\
\cline { 2 - 5 } & 2 & -.500 & .248 & .053 \\
\cline { 2 - 5 } & 4 & $.700^{*}$ & .268 & .014 \\
\cline { 2 - 5 } & 1 & $-2.133^{*}$ & .403 & .000 \\
\cline { 2 - 5 } & 2 & $-1.200^{*}$ & .293 & .000 \\
\hline \multirow{3}{*}{4} & 3 & $-.700^{*}$ & .268 & .014 \\
\hline
\end{tabular}

Table 22: Comparison of mean usual activity score during household work at different follow-up.

\begin{tabular}{|c|c|c|c|c|}
\hline (I) Usual Activity & (2) Usual Activity & Mean Difference (I-J) & Std. Error & Sig. $^{\text {a }}$ \\
\hline \multirow{3}{*}{1} & 2 & $.833^{*}$ & .152 & .000 \\
\cline { 2 - 5 } & 3 & $1.867^{*}$ & .358 & .000 \\
\cline { 2 - 5 } & 4 & $2.733^{*}$ & .386 & .000 \\
\hline \multirow{3}{*}{2} & 1 & $-.833^{*}$ & .152 & .000 \\
\cline { 2 - 5 } & 3 & $1.033^{*}$ & .309 & .002 \\
\hline \multirow{3}{*}{3} & 4 & $1.900^{*}$ & .350 & .000 \\
\cline { 2 - 5 } & 1 & $-1.867^{*}$ & .358 & .000 \\
\cline { 2 - 5 } & 2 & $-1.033^{*}$ & .309 & .002 \\
\hline \multirow{3}{*}{4} & 1 & $.867^{*}$ & .386 & .005 \\
\cline { 2 - 5 } & 2 & $-2.733^{*}$ & .350 & .000 \\
\cline { 2 - 5 } & 3 & $-1.900^{*}$ & .287 & .005 \\
\hline
\end{tabular}

Table 23: Comparison of mean usual activity score during work ( job or everyday work) at different follow-up.

\begin{tabular}{|c|c|c|c|c|}
\hline (I) Usual Activity & (2) Usual Activity & Mean Difference (I-J) & Std. Error & Sig. $^{{ }^{a}}$ \\
\hline \multirow{3}{*}{1} & 2 & $.767^{*}$ & .124 & .000 \\
\cline { 2 - 5 } & 3 & $1.667^{*}$ & .221 & .000 \\
\cline { 2 - 5 } & 4 & $2.667^{*}$ & .333 & .000 \\
\hline \multirow{3}{*}{2} & 1 & $-.767^{*}$ & .124 & .000 \\
\cline { 2 - 5 } & 3 & $.900^{*}$ & .194 & .000 \\
\hline \multirow{3}{*}{3} & 4 & $1.900^{*}$ & .285 & .000 \\
\cline { 2 - 5 } & 1 & $-1.667^{*}$ & .221 & .000 \\
\cline { 2 - 5 } & 2 & $-.900^{*}$ & .194 & .000 \\
\hline
\end{tabular}




\begin{tabular}{|c|c|c|c|c|}
\hline \multirow{3}{*}{4} & 1 & $-2.667^{*}$ & .333 & .000 \\
\cline { 2 - 5 } & 2 & $-1.900^{*}$ & .285 & .000 \\
\cline { 2 - 5 } & 3 & $-1.000^{*}$ & .249 & .000 \\
\hline
\end{tabular}

Table 24: Comparison of mean usual activity score during recreational or sports activity at different follow-up.

\begin{tabular}{|c|c|c|c|c|}
\hline (I) Usual Activity & (2) Usual Activity & Mean Difference (I-J) & Std. Error & Sig. $^{\text {a }}$ \\
\hline \multirow{3}{*}{1} & 2 & $.467^{*}$ & .142 & .003 \\
\cline { 2 - 5 } & 3 & $1.233^{*}$ & .228 & .000 \\
\cline { 2 - 5 } & 4 & $2.167^{*}$ & .437 & .000 \\
\hline \multirow{3}{*}{2} & 1 & $-.467^{*}$ & .142 & .003 \\
\cline { 2 - 5 } & 3 & $.767^{*}$ & .202 & .001 \\
\hline \multirow{3}{*}{3} & 4 & $1.700^{*}$ & .356 & .000 \\
\cline { 2 - 5 } & 1 & $-1.233^{*}$ & .228 & .000 \\
\cline { 2 - 5 } & 2 & $-.767^{*}$ & .202 & .001 \\
\hline \multirow{3}{*}{4} & 4 & $.933^{*}$ & .437 & .006 \\
\cline { 2 - 5 } & 2 & $-2.167^{*}$ & .356 & .000 \\
\cline { 2 - 5 } & 3 & $-1.700^{*}$ & .314 & .006 \\
\hline
\end{tabular}

\section{Discussion}

Purpose of this research was to conclude the comparative effectiveness of Mulligan Mobilization and Therapeutic ultrasound for the treatment of lateral epicondylitis pain regarding reduction of pain and restoration of function. In the present research both of these treatment methods have been used as an intervention to treat the patients with this condition.

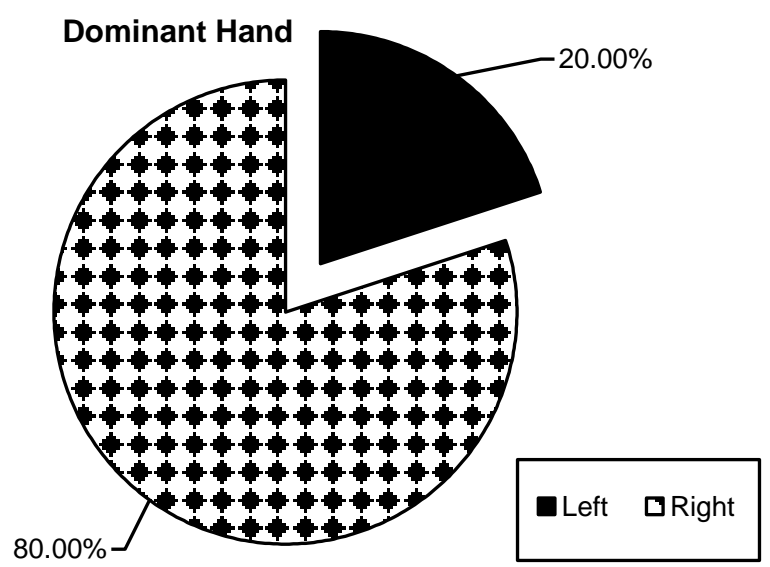

Graph 1: Distribution according to Dominant Hand.

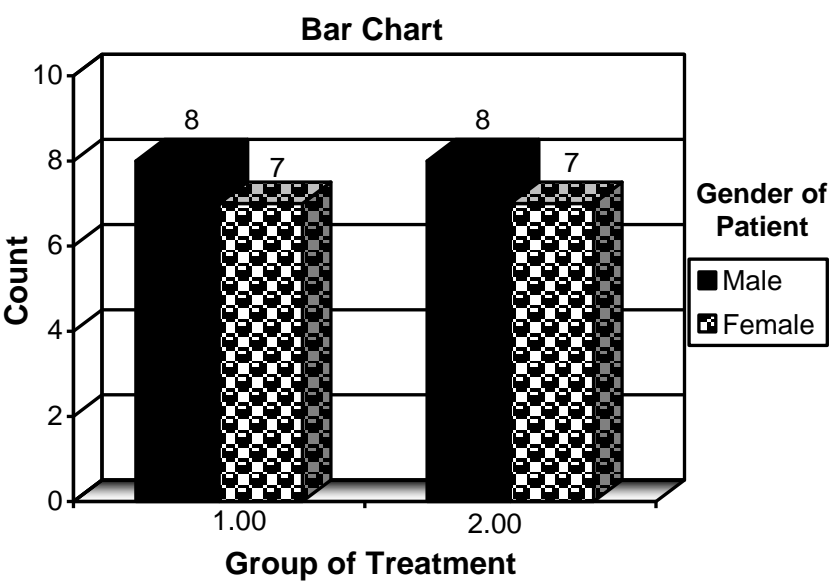

Graph 2: Distribution according to Gender.

Patients with lateral epicondylitis were divided randomly into two groups. In 'group A' Therapeutic Ultrasound was applied to the subjects having LE while in 'group B' technique Mulligan mobilization with Ultrasound Therapy was applied on the elbow.

The questionnaire PRTEE used to assess the patient Total 12 sessions was conducted in duration of 4 weeks after including the patient in study. Four ques- 
tionnaires was asked to fill that was one after every 3 sessions. Overall baseline pain score for repeating arm movement was $6.00 \pm 1.50$, when carrying plastic bag was $7.13 \pm 1.38$ and when pain was at its worse was $8.60 \pm 1.27$. Functional disability score was $6.10 \pm$ 1.62 in cases who were have issue even in turning the knob of door, $7.13 \pm 1.10$ rate was given by those who carry bag, cases who were noted during opening a jar $7.73 \pm 1.11$ score and wring out washing cloth of towel has $5.33 \pm 1.15$ score. Usual activity score at base line on personal activity, household work, work, recreational sporting was $7.03 \pm 1.82,7.00 \pm 1.76$, $7.06 \pm 1.18$ and $5.23 \pm 1.85$ respectively. There was significant decrease in the pain score, functional ability score and usual activity score .Both of group showed decrease in mean score but group B had a significant decrease. So it proved that intervention given to group B was more effective than intervention given to group A.

Our conclusion conform formerly issued trials on patients having lateral epicondylitis pain.Similar study was conducted by Hyunsu Choi (2012) the Experimental group was treated with MWM and Ultrasound Therapy revealed a significant change in the improvements was of $39.13-51.42 \%$, On the other hand the Placebo Control group where without MWM the group did not show any remarkable change in pain and disability with the percentage of $10.71-19.35 .{ }^{14}$ Another identical study in 2009 Owens and Radpasand also showed the similar results in which they concluded the improvement in pain and specific activities in lateral epicondylitis was more than $70 \%$ with Mulligan Mobilization. ${ }^{15}$

Forty subjects were taken and randomly divided in to two groups received Group A was administered with routine Physiotherapy and Group B with routine Physiotherapy and MWM as well by Deepak B Anap (2012). The reevaluation showed more decline in pain and betterment other functional movements in group B which gained the routine Physiotherapy with MWM. ${ }^{15}$

The benefit of the Mulligan mobilization showed a marked decline in pain and PRTEE scores in support of existing result, with respect to the research, the effectiveness of Mulligan's mobilizations techniques have been settled for amending joint function, with a number of hypotheses for its cause and effect. More recent researches have proved further methods that includes the hypoalgesic and sympathetic nervous system excitation effects.

Hence from the accessible studies and the statisti- cal results of data obtained following the treatment deduces that, "Mulligan mobilization technique is a way better than Only Therapeutic ultrasound technique in improving Pain, and disability.

\section{Conclusion and Recommendation}

Combination of Ultrasound Therapy and Mulligan mobilization technique for the cure of lateral epicondylitis has confirmed to be more helpful in aiding pain, and functional impairment in patients having lateral epicondylitis than Ultrasound Therapy without Mulligan Mobilization. The results of this clinical analysis aids in explaining the employment of manual physical therapy in patients with lateral elbow pain. For the true effect of this regimen, future exploration should comprise well - planned randomized control trials with extended treatment durations, longer patient follow-up periods, larger sizes of samples, and self-reported measures of function.

\section{References}

1. Coombes BK, Bisset L, Vicenzino B. Efficacy and safety of corticosteroid injections and other injections for management of tendinopathy: a systematic review of randomised controlled trials. The Lancet. 2010; 376 (9754): 1751-67.

2. Ediz L, Alpayci m. Electrotherapeutic interventions for tennis elbow or lateral epicondylitis: a brief review of the literature. Physics International, 2012; 3 (2): 44.

3. Deepak B. Mobilization with Movement Technique as an Adjunct to Conventional Physiotherapy in Treatment of Chronic Lateral Epicondylitis - A Comparative Study. Journal of Novel Physiotherapies, 2012.

4. Lamba D, Verma S, Basera K, Taragi M, Biswas A. A study to compare the effects of moist heat therapy with ultrasonic therapy and ultrasonic therapy alone in lateral epicondylitis. Indian Journal of. 2012; 6 (2): 41.

5. Jin - Young P. Sports Injuries to the Shoulder and Elbow, 2015.

6. Tosti R, Jennings J, Sewards JM. Lateral epicondylitis of the elbow. The American journal of medicine. 2013; 126 (4): 357. e1-. e6.

7. Ahuja D. Efficacy of Mobilization With Movement (MWM) in Lateral Epicondylalgia: Role of Pain Mechanisms - a Narrative Review. Journal of Physical Therapy (JPT). 2010; 2 (1): 19-34.

8. Bisset LM, Hing W, Vicenzino B. The efficacy of Mobilisations with Movement treatment on musculoskeletal pain: a systematic review and meta - analysis, 2011. 
9. Bhardwaj P, Dhawan A. The relative efficacy of mobilization with movement versus Cyriax physiotherapy in the treatment of lateral epicondylitis. Indian Journal of Physiotherapy and Occupational Therapy - An International Journal, 2011; 5 (1): 142-6.

10. Janikowska K, Fidut J. The role of Mulligan Therapy Concept in treatment of "tennis elbow" symptoms. New Approaches in Joints Pain, 2013: 20.

11. Dingemanse R, Randsdorp M, Koes BW, Huisstede BM. Evidence for the effectiveness of electrophysical modalities for treatment of medial and lateral epicondylitis: a systematic review. British journal of sports medicine, 2014; 48 (12): 957-65.

12. Tsai W-C, Tang S-T, Liang F-C. Effect of therapeutic ultrasound on tendons. American Journal of Physical Medicine and Rehabilitation, 2011; 90 (12): 1068-73.

13. de Brito Vieira, W. H., Aguiar, K. A., da Silva, K. M., Canela, P. M., da Silva, F. S. and Abreu, B. J. Over- view of ultrasound usage trends in orthopedic and sports physiotherapy. Critical ultrasound journal, 2012; 4 (1): 11 .

13. Kim, L. J., Choi, H. and Moon, D. Improvement of Pain and Functional Activities in Patients with Lateral Epicondylitis of the Elbow by Mobilization with Movement: a Randomized, Placebo - Controlled Pilot Study. Journal of Physical Therapy Science, 2012; 24 (9): 787790.

14. Radpasand, M. Combination of manipulation, exercise, and physical therapy for the treatment of a 57-year-old woman with lateral epicondylitis. Journal of manipulative and Physiological Therapeutics, 2009; 32 (2): 166-172.

15. Deepak, B. Mobilization with Movement Technique as an Adjunct to Conventional Physiotherapy in Treatment of Chronic Lateral Epicondylitis - A Comparative Study. Journal of Novel Physiotherapies, 2012. 